J. Korean Math. Soc. 47 (2010), No. 2, pp. 289-297

DOI 10.4134/JKMS.2010.47.2.289

\title{
EQUIDIMENSIONAL SYMMETRIC ALGEBRAS
}

\author{
Agustín Marcelo, FÉlix Marcelo, and CÉsar Rodríguez
}

AbStract. By using as main tool the theory of prime submodules this article is devoted to describing the structure of the minimal prime ideals of the equidimensional symmetric algebra of a finitely generated module.

\section{Introduction}

In this paper we obtain two basic results concerning equidimensional symmetric algebras. By using the theory of prime submodules we first characterize when the ideal generated by a submodule of a free module in the symmetric algebra is equidimensional. The next section is devoted to determining minimal components of equidimensional symmetric algebras. Probably the most outstanding result about this question was obtained by Huneke and Rossi in [1, Section 3]. These authors showed, among other results, that if $N$ is a finitelygenerated module over a commutative Noetherian ring, then the symmetric algebra of $N, S(N)$, can have arbitrarily large number of minimal components and they tried to identify the prime ideals of $R$ which are the contraction of a minimal prime of $S(N)$.

Let $R$ be a universally catenary Noetherian domain and let $N$ be a finitely generated $R$-module such that $S(N)$ is equidimensional. If $\mathfrak{p}$ is a prime ideal of $R$, then the least number of generators of $N_{\mathfrak{p}}$ is denoted by $\nu\left(N_{\mathfrak{p}}\right)$. Let $f: \operatorname{Spec} S(N) \rightarrow$ Spec $R$ denote the induced natural map. Then, given $\mathfrak{p} \in$ Spec $R$, our basic purpose is to prove that in the $f^{-1}(\mathfrak{p})$ there is a minimal prime ideal of $S(N)$ if and only if $\nu\left(N_{\mathfrak{p}}\right)-h t \mathfrak{p}=\operatorname{rank} N$; see Theorem 11 below. If $\mathfrak{p}$ fulfils this condition, then there exists a unique minimal prime ideal of $S(N)$ in $f^{-1}(\mathfrak{p})$, denoted by $\mathcal{E}_{\mathfrak{p}(0)}$ and defined as

$$
\mathcal{E}_{\mathfrak{p}(0)}=\{b \in S(N): a b \in \mathfrak{p} \cdot S(N) \text { for some } a \in R \backslash \mathfrak{p}\} .
$$

We finish this article by using the primary decomposition of $(0) \subset S(N)$ in order to solve an open problem of [1] about the set of prime ideals of $R$ which are contractions of minimal prime ideals of $S(N)$.

Received May 15, 2008.

2000 Mathematics Subject Classification. 15A69, 16W50, 16D25.

Key words and phrases. prime submodules, symmetric algebra, minimal components. 


\section{Preliminaries}

Let $R$ be a commutative Noetherian ring with identity and let $N$ be a finitely generated $R$-module. Recall that a proper submodule $P$ of $N$ is said to be a prime (resp. primary) submodule if for every $a \in R$, the induced homothety $N / P \stackrel{\cdot a}{\longrightarrow} N / P$ is either injective or null (resp. nilpotent). In light of this definition, it turns out that if $P$ is a prime (resp. primary) submodule of $N$, then the set of homotheties of $R$ vanishing on $N / P$, i.e.,

$$
(P: N)=\{a \in R / a N \subseteq P\}=\operatorname{Ann}(N / P)
$$

is a prime (resp. primary) ideal of $R$. Furthermore, if $P$ is a primary submodule of $N$, the radical of the primary ideal $(P: N)$, denoted by $\sqrt{(P: N)}$, is a prime ideal of $R$ formed by all nilpotent homotheties of $R$ on $N / P$, i.e., $\sqrt{(P: N)}=\left\{a \in R / a^{n} N \subseteq P\right.$ for some $\left.n>0\right\}$. Thus if $P$ is a prime submodule of $N$ with $\mathfrak{p}=(P: N)$ we shall call $P$ a $\mathfrak{p}$-prime submodule and if $P$ is a primary submodule of $N$ being $\mathfrak{p}=\sqrt{(P: N)}$ we will say that $P$ is a $\mathfrak{p}$-primary submodule. Note that a $\mathfrak{p}$-primary submodule $P$ of $N$ is $\mathfrak{p}$-prime if and only if $(P: N)=\mathfrak{p} \in \operatorname{Spec} R$.

Let $L$ be a proper submodule of a $R$-module $N$. Given a prime ideal $\mathfrak{p}$ of $R$, we will denote by $\mathfrak{p}(L)$ the following submodule of $N$ :

$$
\mathfrak{p}(L)=\{n \in N: \text { an } \in L+\mathfrak{p} N \text { for some } a \in R \backslash \mathfrak{p}\} .
$$

With the above notations, it is easy to see that either $\mathfrak{p}(L)=N$ or $\mathfrak{p}(L)$ is a $\mathfrak{p}$-prime submodule of $N$, which is contained in every $\mathfrak{p}$-prime submodule of $N$ containing $L$.

On the other hand, for every $\mathfrak{p}$-prime submodule $P$ of $N$ there exists a prime ideal $\mathcal{E}_{P}$ of the symmetric algebra of $N$, called the expansion of $P$ (see [2, Section 3]) and defined to be the following set:

$$
\mathcal{E}_{P}=\{b \in S(N): a b \in(\mathfrak{p}, P) \cdot S(N) \text { for some } a \in R \backslash \mathfrak{p}\},
$$

where we use the identifications $R \simeq S_{0}(N)$ and $N \simeq S_{1}(N)$. In particular, the ideal $\mathcal{E}_{(0)}$ coincides with the $R$-torsion ideal $T$ of $S(N)$ introduced in [4, p. 3].

Proposition 1. Let $(R, \mathfrak{p})$ be a Noetherian local ring, let $F$ be a free $R$-module of rank $n$ and let $M$ be a proper submodule of $F$. Then either $\mathfrak{p}(M)=\mathfrak{p} F$ or $\mathfrak{p}(M)=\mathfrak{p} F+\left(e_{1}, \ldots, e_{h}\right)$, where $e_{1}, \ldots, e_{h}$ form a part of a basis of $F$.

Proof. From the definition of $\mathfrak{p}(M)$ and taking into account that every element of $R-\mathfrak{p}$ is invertible it follows that $\mathfrak{p}(M)=M+\mathfrak{p} F$. If $\mathfrak{p}(M)=\mathfrak{p} F$, we conclude. Assume $\mathfrak{p}(M) \neq \mathfrak{p} F$. Since $\mathfrak{p} F \subset \mathfrak{p}(M)$, by applying Nakayama's lemma there exist elements $e_{1}, \ldots, e_{h}$ in $M+\mathfrak{p} F$ such that $e_{1}, e_{2}, \ldots, e_{h}$ form a part of a basis for $F$ and $M+\mathfrak{p} F=\mathfrak{p} F+\left(e_{1}, e_{2}, \ldots, e_{h}\right)$.

Remark 2. From now on, we assume that $h$ is the largest possible value. 
Proposition 3. Let $(R, \mathfrak{p}), F$ and $M$ be as above. Then, either

$$
\mathcal{E}_{\mathfrak{p}(M)}=\mathfrak{p} S(F)
$$

or

$$
\mathcal{E}_{\mathfrak{p}(M)}=\mathfrak{p} S(F)+\left(x_{1}, x_{2}, \ldots, x_{h}\right) S(F),
$$

where $S(F)=R\left[x_{1}, x_{2}, \ldots, x_{h}, x_{h+1}, \ldots, x_{n}\right]$.

Proof. By reasoning in a similar way as above we have

$$
\mathcal{E}_{\mathfrak{p}(M)}=\mathfrak{p} S(F)+M \cdot S(F) .
$$

If $\mathcal{E}_{\mathfrak{p}(M)}=\mathfrak{p} S(F)$, then we are done. Otherwise, by applying the above proposition and identifying $F=S_{1}(F)$ it follows that $\mathcal{E}_{\mathfrak{p}(M)}=\mathfrak{p} S(F)+$ $\left(x_{1}, \ldots, x_{h}\right) S(F)$.

Proposition 4. Let $(R, \mathfrak{p}), F$, and $M$ be as above. Then, either

$$
\operatorname{tr} . \operatorname{deg}_{k(\mathfrak{p})} k\left(\mathcal{E}_{\mathfrak{p}(M)}\right)=n \text { if } \mathcal{E}_{\mathfrak{p}(M)}=\mathfrak{p} S(F),
$$

or

$$
\operatorname{tr} . \operatorname{deg}_{k(\mathfrak{p})} k\left(\mathcal{E}_{\mathfrak{p}(M)}\right)=n-h \text { if } \mathcal{E}_{\mathfrak{p}(M)}=\mathfrak{p} S(F)+\left(x_{1}, \ldots, x_{h}\right) S(F) .
$$

Proof. If $\mathcal{E}_{\mathfrak{p}(M)}=\mathfrak{p} S(F)$ the first equality is obvious. Assume

$$
\mathcal{E}_{\mathfrak{p}(M)}=\mathfrak{p} S(F)+\left(x_{1}, \ldots, x_{h}\right) S(F) .
$$

It is not difficult to see that $\overline{S(F)}=S(F) / \mathcal{E}_{\mathfrak{p}(M)}$ is the polynomial ring

$$
R / \mathfrak{p}\left[\overline{x_{h+1}}, \ldots, \overline{x_{n}}\right] \text {, }
$$

where

$$
\begin{aligned}
k(\mathfrak{p}) & =R / \mathfrak{p}, \\
k\left(\mathcal{E}_{\mathfrak{p}(M)}\right) & =\overline{S(F)}_{(0)} .
\end{aligned}
$$

Hence the desired result follows.

Again, let $(R, \mathfrak{p}), F$ and $M$ be as above. Let us consider the short exact sequence $0 \longrightarrow M \longrightarrow F \stackrel{\pi}{\longrightarrow} N \longrightarrow 0$ and let $\nu(N)$ be the minimal number of generators of $N$. As we have seen, if $M+\mathfrak{p} F=\mathfrak{p} F+\left(e_{1}, e_{2}, \ldots, e_{h}\right)$, then there exists a basis $\left\{e_{1}, e_{2}, \ldots, e_{h}, e_{h+1}, \ldots, e_{n}\right\}$ of $F$. Thus, by Nakayama's lemma, $\nu(N)=n-h$.

Proposition 5. With the same hypotheses and notations as above,

$$
\text { tr. } \operatorname{deg}_{k(\mathfrak{p})} k\left(\mathcal{E}_{\mathfrak{p}(M)}\right)=\nu(N) .
$$

Proof. From the preceding proposition we have tr. $\operatorname{deg}_{k(\mathfrak{p})} k\left(\mathcal{E}_{\mathfrak{p}(M)}\right)=n-h$. On the other hand, as we have just point out $\nu(N)=n-h$ and the result is proved. 


\section{Equidimensional ideals}

Assumption 6. Let $R$ be a Noetherian domain, let $F$ be a free $R$-module of finite rank, and let $S(F)$ be the symmetric algebra of $F$. In what follows, we assume that every irredundant chain of prime ideals of $S(F)$ has the same length.

Remark 7. Let $M$ be a submodule of $F$ and denote by $M \cdot S(F)$ the ideal generated by $M$ in $S(F)$. As is well known, the ideal $M \cdot S(F)$ is said to be equidimensional if all its minimal prime ideals have the same codimension. These ideals are interesting because the quotient $S(F) / M \cdot S(F)$ is an equidimensional symmetric algebra.

Proposition 8. Every minimal prime ideal over $M \cdot S(F)$ is the expansion $\mathcal{E}_{\mathfrak{p}(M)}$ for some $\mathfrak{p} \in \operatorname{Spec} R$.

Proof. Let $I \in \operatorname{Spec} S(F)$ be a minimal prime ideal over $M \cdot S(F)$ and set $\mathfrak{p}=I \cap R$. Then it is easily shown that $P=I \cap F$ is a $\mathfrak{p}$-prime submodule containing $M$. Hence $\mathfrak{p}(M) \subseteq P$ and we have $\mathcal{E}_{\mathfrak{p}(M)} \subseteq \mathcal{E}_{P} \subseteq I$. Since $I$ is a minimal ideal over $M \cdot S(N)$ and $M \cdot S(N) \subseteq \mathcal{E}_{\mathfrak{p}(M)}$ it is deduced that $\mathcal{E}_{\mathfrak{p}(M)}=I$ and the desired equality follows.

Proposition 9. With the notations of Proposition 5, let $M \subset F$ be a submodule and let $r_{\mathfrak{p}}(M)$ be the greatest rank of a free direct summand of $F_{\mathfrak{p}}$ contained in $M_{\mathfrak{p}}, \mathfrak{p} \in \operatorname{Spec} R$. Then,

$$
h t \mathcal{E}_{\mathfrak{p}(M)}=h t \mathfrak{p}+r_{\mathfrak{p}}(M) .
$$

Proof. From the properties of localization it follows

$$
h t \mathcal{E}_{\mathfrak{p}(M)}=\left(h t \mathcal{E}_{\mathfrak{p}(M)}\right)_{\mathfrak{p}} .
$$

On the other hand, we have

$$
M_{\mathfrak{p}}=M_{\mathfrak{p}}^{\prime} \oplus l_{\mathfrak{p}}(M),
$$

where $l_{\mathfrak{p}}(M)$ is a direct summand of $F_{\mathfrak{p}}$ contained in $M_{p}$ of $\operatorname{rank} r_{\mathfrak{p}}(M)$ and $M_{\mathfrak{p}}^{\prime}$ is a submodule of $M_{\mathfrak{p}}$. This implies that $M_{\mathfrak{p}}^{\prime} \subseteq \mathfrak{p} F_{\mathfrak{p}}$ since if an element $m^{\prime} \in M_{\mathfrak{p}}^{\prime}$ is not included in $\mathfrak{p} F_{\mathfrak{p}}$, then $\left(m^{\prime}\right)$ would be a direct summand of $F_{\mathfrak{p}}$ as follows from Nakayama's lemma. Hence

$$
M_{\mathfrak{p}}=M_{\mathfrak{p}}^{\prime \prime} \oplus\left(m^{\prime}\right) \oplus l_{\mathfrak{p}}(M),
$$

thus contradicting the greatest rank of $l_{\mathfrak{p}}(M)$. Now it is not difficult to see that

$$
\mathfrak{p}\left(M_{\mathfrak{p}}\right)=\mathfrak{p} F_{p} \oplus l_{\mathfrak{p}}(M) .
$$

By identifying, as usual, $F$ to $S_{1}(F)$ it follows that $\mathfrak{p} F_{p} \oplus l_{\mathfrak{p}}(M)$ generates an ideal of $S(F)_{\mathfrak{p}}$ whose height is just $h t \mathfrak{p}+r_{\mathfrak{p}}(M)$. Again by properties of localization we obtain that the precedent height coincides with the height of the ideal $\mathcal{E}_{\mathfrak{p}(M)}$, and so the proof is completed. 
Before passing to the statement of our next result, we need to prove the following lemma:

Lemma 10. $\mathcal{E}_{0(M)}$ is a minimal prime ideal over $M \cdot S(M)$ such that

$$
h t \mathcal{E}_{0(M)}=\operatorname{rank}(M) .
$$

Proof. First assume that $\mathcal{E}_{0(M)}$ is not minimal over $M \cdot S(M)$. Let $I$ be a prime ideal of $S(F)$ such that

$$
M \cdot S(F) \subseteq I \subsetneq \mathcal{E}_{0(M)}
$$

If $I \cap R=(0)$ it is not difficult to see that $I=\mathcal{E}_{0(M)}$, contrary to the initial assumption. Thus let $I \cap R=\mathfrak{p} \neq(0)$. Since $\mathcal{E}_{0(M)} \cap R=(0)$, after localizing $S(F)$ by the multiplicative set $S=R-(0)$ it turns out that $\left(\mathcal{E}_{0(M)}\right)_{(0)}$ is a proper ideal of $S(F)_{(0)}$ and the same happens for the ideal $I_{(0)}$ since $I \subsetneq \mathcal{E}_{0(M)}$. But in $S(F)_{(0)}$ we have $\mathfrak{p}_{(0)}=R_{(0)}$ it follows that $I_{(0)}$ contains the identity element. Therefore $I_{(0)}=S(F)_{(0)}$ which leads us to a contradiction. Hence $I=\mathcal{E}_{0(M)}$. On the other hand, $S(F)_{(0)}$ is a polynomial ring over the field $R_{(0)}$ in which $M \cdot S(F)_{(0)}$ is a prime ideal whose height is just $\operatorname{rank}(M)$. Finally, since $M \cdot S(F)_{(0)}=\left(\mathcal{E}_{0(M)}\right){ }_{(0)}$ we deduced the desired result taking into account that $h t \mathcal{E}_{0(M)}=h t\left(\mathcal{E}_{0(M)}\right)_{(0)}$.

Theorem 11. With the notations of Proposition 5, the following conditions are equivalent:

(1) $M \cdot S(F)$ is an equidimensional ideal.

(2) If $\mathcal{E}_{\mathfrak{p}(M)}$ is a minimal prime ideal over $M \cdot S(F)$, then

$$
h t \mathfrak{p}+r_{\mathfrak{p}}(M)=\operatorname{rank}(M) .
$$

Proof. Assume $M \cdot S(F)$ is an equidimensional ideal and let $I$ be a minimal prime ideal over $M \cdot S(M)$. By virtue of Proposition 5 the ideal $I$ is the expansion of a prime submodule $\mathfrak{p}(M)$ where by definition we have

$$
I \cap F=\mathfrak{p}(M) \text { and } I=\mathcal{E}_{\mathfrak{p}(M)} .
$$

By applying Proposition 6 it now follows that

$$
h t I=h t \mathfrak{p}+r_{\mathfrak{p}}(M) .
$$

On the other hand, by localizing $M \cdot S(F)$ in the generic point of $R$ and by contracting this localization to $S(F)$ we obtain just the ideal $\mathcal{E}_{0(M)}$.

Using now the precedent lemma and the hypothesis of equidimensionality of $M \cdot S(F)$, we deduce that

$$
h t \mathfrak{p}+r_{\mathfrak{p}}(M)=\operatorname{rank}(M) .
$$

Let us assume now that for every minimal prime ideal $\mathcal{E}_{\mathfrak{p}(M)}$ over $M \cdot S(F)$ is $h t \mathfrak{p}+r_{\mathfrak{p}}(M)=\operatorname{rank}(M)$. Clearly in this case $M \cdot S(F)$ is equidimensional and we can conclude. 


\section{Minimal components of $S(N)$}

We first need the following

Lemma 12. Let $R$ be a universally catenary Noetherian domain, let $\mathfrak{p}$ be a prime ideal of $R$, let $N$ be a finitely generated $R$-module and let

$$
0 \longrightarrow M \cdot S(F) \longrightarrow S(F) \stackrel{\lambda}{\longrightarrow} S(N) \longrightarrow 0
$$

be the exact sequence induced from the exact sequence of $R$-modules,

$$
0 \longrightarrow M \longrightarrow F \stackrel{\pi}{\longrightarrow} N \longrightarrow 0,
$$

in which $F$ is free. Then, $\mathcal{E}_{\mathfrak{p}(0)}$ is a minimal prime ideal of $S(N)$ if and only if $\mathcal{E}_{\mathfrak{p}(M)}$ is a minimal prime ideal over $M \cdot S(F)$.

Proof. Assume that $\mathcal{E}_{\mathfrak{p}(0)}$ is a minimal prime ideal of $S(N)$. In this case, we can easily see that $\lambda^{-1}\left(\mathcal{E}_{\mathfrak{p}(0)}\right)$ is a minimal prime ideal over $M \cdot S(F)$ and $\lambda^{-1} \mathcal{E}_{\mathfrak{p}(0)}=\mathcal{E}_{\mathfrak{p}(M)}$.

Conversely, if $\mathcal{E}_{\mathfrak{p}(M)}$ is a minimal prime ideal of $S(F)$ over $M \cdot S(F)$, then $\lambda\left(\mathcal{E}_{\mathfrak{p}(M)}\right)=\mathcal{E}_{\mathfrak{p}(0)}$ is also a minimal prime in $S(N)$.

Theorem 13. With the same notations and assumptions as in the previous lemma, $\mathcal{E}_{\mathfrak{p}(0)}$ is a minimal prime ideal of $S(N)$ if and only if $\operatorname{rank} N=\nu\left(N_{\mathfrak{p}}\right)-$ $h t \mathfrak{p}$.

Proof. Let $T=T(S(N))$ be the torsion $R$-module of $S(N)$. By [1, p. 201] $T$ is a minimal prime ideal of $S(N)$. Therefore $\lambda^{-1}(T)$ is a prime ideal of $S(F)$ minimal over $M \cdot S(F)$. Moreover, we have $\lambda^{-1}(T)=\mathcal{E}_{0(M)}$, where $\mathcal{E}_{0(M)}$ is the expansion of the 0 -prime submodule $0(M)$, i.e.,

$$
\mathcal{E}_{0(M)}=\{b \in S(F): a b \in M \cdot S(F) \text { for some } a \neq 0\} .
$$

$\Longrightarrow$ Assume now $\mathcal{E}_{\mathfrak{p}(M)}$ is a minimal prime ideal over $M \cdot S(F)$. Taking into account that $S(F)$ is a catenary ring because $R$ is universally catenary and by hypothesis $S(N)$ is equidimensional, it is not difficult to see that all minimal prime ideals over $M \cdot S(F)$ have the same height. Then $h t \mathcal{E}_{\mathfrak{p}(M)}=h t \mathcal{E}_{0(M)}$ (see [3, p. 118]). On the other hand, by applying [3, Theorem 15.5, p. 118] we obtain

$$
h t \mathcal{E}_{0(M)}=\operatorname{tr} . \operatorname{deg}_{k(0)} S(F)_{(0)}-\operatorname{tr} . \operatorname{deg}_{k(0)} k\left(\mathcal{E}_{0(M)}\right),
$$

where $k(0)$ is the field of fractions of $R$.

Next, from Proposition 4, we have

$$
\text { tr. } \operatorname{deg}_{k(0)} k\left(\mathcal{E}_{0(M)}\right)=\nu\left(N_{(0)}\right)=\operatorname{rank} N .
$$

Hence

$$
h t \mathcal{E}_{0(M)}=\operatorname{tr} . \operatorname{deg}_{k(0)} S(F)_{(0)}-\operatorname{rank} N .
$$

Again by [3, p. 118] we have

$$
h t \mathcal{E}_{\mathfrak{p}(M)}=\operatorname{tr} . \operatorname{deg}_{k(0)} S(F)_{(0)}+h t \mathfrak{p}-\operatorname{tr} . \operatorname{deg}_{k(\mathfrak{p})} k\left(\mathcal{E}_{\mathfrak{p}(M)}\right),
$$


thus

$$
h t \mathcal{E}_{\mathfrak{p}(M)}=\operatorname{tr} . \operatorname{deg}_{k(0)} S(F)_{(0)}+h t \mathfrak{p}-\nu\left(N_{\mathfrak{p}}\right)
$$

which implies that $\operatorname{rank} N=\nu\left(N_{\mathfrak{p}}\right)-h t \mathfrak{p}$.

$\Longleftarrow)$ Conversely, suppose that $\operatorname{rank} N=\nu\left(N_{\mathfrak{p}}\right)-h t \mathfrak{p}$. By applying [3, p. 118] and the above theorem we obtain

$$
\begin{aligned}
h t \mathcal{E}_{\mathfrak{p}(M)} & =h t \mathfrak{p}+\text { tr. } \operatorname{deg}_{k(0)} S(F)_{(0)}-\operatorname{tr} \cdot \operatorname{deg}_{k(\mathfrak{p})} k\left(\mathcal{E}_{0(M)}\right) \\
& =h t \mathfrak{p}+\text { tr. } \operatorname{deg}_{k(0)} S(F)_{(0)}-\nu\left(N_{\mathfrak{p}}\right) .
\end{aligned}
$$

On the other hand,

$$
\begin{aligned}
h t \mathcal{E}_{0(M)} & =\operatorname{tr} \cdot \operatorname{deg}_{k(0)} S(F)_{(0)}-\operatorname{tr} \cdot \operatorname{deg}_{k(0)} k\left(\mathcal{E}_{M}\right) \\
& =\operatorname{tr} \cdot \operatorname{deg}_{k(0)} S(F)_{(0)}-\nu\left(N_{(0)}\right) \\
& =\operatorname{tr} \cdot \operatorname{deg}_{k(0)} S(F)_{(0)}-\operatorname{rank} N .
\end{aligned}
$$

Then, by virtue of hypothesis $h t \mathcal{E}_{\mathfrak{p}(M)}=h t \mathcal{E}_{0(M)}$. By using the fact that $S(F)$ is a catenarian ring it is deduced that $\mathcal{E}_{\mathfrak{p}(M)}$ is a minimal prime ideal over $M \cdot S(F)$ in $S(F)$ since $\mathcal{E}_{\mathfrak{p}(0)}$ is minimal in $S(N)$.

\section{Induced primary decompositions}

Let $R, N$, and $S(N)$ be as in Theorem 9 and let $R \simeq S_{0}(N), N \simeq S_{1}(N)$ be the traditional identifications. Let

$$
(0)=J_{1} \cap \cdots \cap J_{r}
$$

be a primary decomposition of $(0) \in S(N)$. It is not difficult to see that

$$
Q_{i}=J_{i} \cap N \quad i=1, \ldots, r,
$$

is a primary submodule of $N$ and also that

$$
(0)=Q_{1} \cap \cdots \cap Q_{r}
$$

is a primary decomposition of $(0) \in N$.

Let $\Lambda$ be the set of $\operatorname{Spec} R$ such that $\mathfrak{p} \in \Lambda$ if there exists a minimal prime $P \in \operatorname{Spec} S(N)$ with $P \cap R=\mathfrak{p}$.

Theorem 14. Let

$$
(0)=Q_{1} \cap \cdots \cap Q_{k} \cap \cdots \cap Q_{r}
$$

be the primary decomposition above, where

$$
\operatorname{rank} N=\nu\left(N_{\mathfrak{p}_{i}}\right)-h t \mathfrak{p}, \quad i=1, \ldots, k .
$$

Let $\mathfrak{p}_{i}=\operatorname{Ann}\left(N / Q_{i}\right), i=1, \ldots, k, \ldots, r$. Then,

$$
\Lambda=\left\{\mathfrak{p}_{1}, \ldots, \mathfrak{p}_{k}\right\} \text {. }
$$


Proof. By using Theorem 9, it follows that $\mathcal{E}_{\mathfrak{p}_{i}(0)}$ is a minimal prime ideal of $S(N)$ for $i=1, \ldots, k$.

Conversely, if $P$ is a minimal prime ideal of $S(N)$, it is well known that $P$ is the radical of $J_{i}$ for some $i \in\{1, \ldots, k, \ldots, r\}$. If $\mathfrak{p}=P \cap R$, again by Theorem 9 , we have $\operatorname{rank} N=\nu\left(N_{\mathfrak{p}}\right)-h t \mathfrak{p}$. Therefore, $\mathfrak{p}$ is one of the ideals $\mathfrak{p}_{1}, \ldots, \mathfrak{p}_{k}$.

The induced primary decomposition of the theorem above is not minimal in general, but it contains all the information that we need to get the minimal prime ideals of $S(N)$. A minimal decomposition of $(0) \in N$ does not always contain such information as we shall see below.

Example 15. Let $R$ be the local ring in the origin of $k[x, y, z, t]$, where $k$ is a field. Let $F$ be a free $R$-module of rank four and let $e_{1}, e_{2}, e_{3}, e_{4}$ be a basis of $F$. Let $h=x e_{1}+y e_{2}, g=x e_{3}+y e_{4}$ two elements of $F$, and let $E=(h, g)$ be the submodule of $F$ generated by $h, g$. Finally, let $M$ be the submodule of $F$ such that $m \in M$ if there exists $a \in R, a \neq 0$ with $a m \in E$. It is not difficult to see that $N=F / M$ is a torsion-free $R$-module of rank two.

Let $S(N)$ be the symmetric algebra of $N$ and let $\mathfrak{p}_{1}=(0), \mathfrak{p}_{2}=(x, y)$. Since $R$ is a domain we have

$$
\begin{gathered}
\left.\operatorname{rank} N=\operatorname{dim}_{R_{(0)}} N_{(0)}=\nu\left(N_{(0)}\right)\right)=2, \\
\operatorname{rank} N=\nu\left(N_{\mathfrak{p}}\right)-h t \mathfrak{p}=4-2,
\end{gathered}
$$

and by using Theorem 9 , we see that $\mathfrak{p}_{1}, \mathfrak{p}_{2}$ give rises to two minimal prime ideals $P_{1}, P_{2}$ of $S(N)$ and these are the only ones. Let

$$
\text { (0) }=J_{1} \cap J_{2} \cap \cdots \cap J_{r}
$$

be a primary decomposition of $(0) \in S(N)$. Without lost of generality we may assume that $P_{1}, P_{2}$ are the radicals of $J_{1}, J_{2}$.

The above decomposition induces another one,

$$
(0)=Q_{1} \cap Q_{2} \cap \cdots \cap Q_{r}
$$

of $(0) \in N$. This new decomposition is clearly redundant since $(0)$ is a primary submodule of $N$ because $N=N /(0)$ is torsion-free. Nevertheless, this decomposition contains all the information about the set $\Lambda$.

Acknowledgement. The authors thank Professor W. Vasconcelos for his valuable comments in the preparation of this article. Supported by Ministerio de Ciencia y Tecnología of Spain, under grant BFM2001-2718.

\section{References}

[1] C. Huneke and M. Rossi, The dimension and components of symmetric algebras, J. Algebra 98 (1986), no. 1, 200-210.

[2] A. Marcelo and C. Rodríguez, Radicals of submodules and symmetric algebra, Comm. Algebra 28 (2000), no. 10, 4611-4617.

[3] H. Matsumura, Commutative Ring Theory, Cambridge University Press, Cambridge, 1986. 
[4] W. Vasconcelos, Arithmetic of Blowup Algebras, Cambridge University Press, Cambridge, 1994.

Agustín Marcelo

Dpto. Matemáticas

Universidad de Las Palmas de Gran Canaria

CAMPus DE TAFIRA

35017 Las Palmas de Gran Canaria, Spain

E-mail address: amarcelo@dma.ulpgc.es

Félix Marcelo

Dpto. Matemáticas

Universidad de Las Palmas de Gran Canaria

CAMPus de TAFira

35017 Las Palmas de Gran Canaria, Spain

E-mail address: fjmarcelo@gmail.com

CÉsar Rodríguez

Dpto. Matemáticas

Universidad de Las Palmas de Gran Canaria

CAMPus de TAFira

35017 Las Palmas de Gran Canaria, Spain

E-mail address: cesar@dma.ulpgc.es 